\title{
PARAMETRIC PROBLEM OF COMPLETELY GENERALIZED QUASI-VARIATIONAL INEQUALITIES
}

\author{
SALAHUDDIN, M. K. AHMAD, AND A. H. SIDDIQI
}

Received 29 August 2004; Revised 27 January 2005; Accepted 29 June 2005

This paper is devoted to the study of behaviour and sensitivity analysis of the solution for a class of parametric problem of completely generalized quasi-variational inequalities.

Copyright (c) 2006 Salahuddin et al. This is an open access article distributed under the Creative Commons Attribution License, which permits unrestricted use, distribution, and reproduction in any medium, provided the original work is properly cited.

\section{Introduction}

Sensitivity analysis of solutions for variational inequalities with single-valued mappings has been studied by many authors with different techniques in finite dimensional spaces and Hilbert spaces $[3,4,7,11,14]$. Robinson [10] has dealt with the sensitivity analysis of solutions for the classical variational inequalities over polyhedral convex sets in finite dimensional spaces.

In this paper, we study the behaviour and sensitivity analysis of solutions for a class of parametric problem of completely generalized quasi-variational inequalities with setvalued mappings without the differentiability assumptions.

\section{Preliminaries}

Let $H$ be a real Hilbert space with $\|x\|^{2}=\langle x, x\rangle, 2^{H}$ the family of all nonempty bounded subsets of $H$ and $C(H)$ the family of all nonempty compact subsets of $H$. Let $\delta: 2^{H} \rightarrow$ $[0, \infty)$ be defined by

$$
\delta(A, B)=\sup \{\|a-b\|: a \in A, b \in B\}, \quad \forall A, B \in 2^{H},
$$

and let $\tilde{H}: C(H) \rightarrow[0, \infty)$ be defined by

$$
\tilde{H}(A, B)=\max \left\{\sup _{x \in A} d(x, B), \sup _{y \in B} d(A, y)\right\}, \quad \forall A, B \in C(H),
$$


2 Parametric problem of quasi-variational inequalities

where

$$
d(x, B)=\inf _{y \in B}\|x-y\| .
$$

Then, $\left(2^{H}, \delta\right)$ and $(C(H), \tilde{H})$ are complete metric spaces, $\tilde{H}$ is the Hausdorff metric on $C(H)$.

We now consider the parametric problem of completely generalized quasi-variational inequalities. Let $\Omega$ be a nonempty open subset of $H$ in which the parameter $\lambda$ takes values and $K: H \times \Omega \rightarrow 2^{H}$ set-valued mapping with nonempty closed convex valued. Let $A, R, T: H \times \Omega \rightarrow 2^{H}$ be the set-valued mappings and $p, f, g, G: H \times \Omega \rightarrow H$ the singlevalued mappings. For each fixed $\lambda \in \Omega$, we write $G_{\lambda}(x)=G(x, \lambda), u_{\lambda}(x)=u(x, \lambda)$ unless otherwise specified. The parametric problem of completely generalized quasi-variational inequality (PPCGQVI) consists in finding $x \in H, u_{\lambda}(x) \in A_{\lambda}(x), w_{\lambda}(x) \in R_{\lambda}(x), z_{\lambda}(x) \in$ $T_{\lambda}(x)$ such that $G_{\lambda}(x) \in K_{\lambda}(x)$ and

$$
\left\langle p_{\lambda}\left(u_{\lambda}(x)\right)-\left(f_{\lambda}\left(w_{\lambda}(x)\right)-g_{\lambda}\left(z_{\lambda}(x)\right)\right), y-G_{\lambda}(x)\right\rangle \geq 0, \quad \forall y \in K_{\lambda}(x) .
$$

In many important applications, $K_{\lambda}(x)$ has the form

$$
K_{\lambda}(x)=m(x)+K_{\lambda}, \quad \forall(x, \lambda) \in H \times \Omega,
$$

where $m: H \rightarrow H$ and $\left\{K_{\lambda}: \lambda \in \Omega\right\}$ is a family of nonempty closed and convex subsets of $H$, see, for example, [13] and the references therein.

For each $\lambda \in \Omega$, let $S(\lambda)$ denote the set of solutions to the problem (2.4). For some $\bar{\lambda} \in \Omega$, we fix those conditions under which for each $\lambda$ in a neighborhood ( $\operatorname{say} N(\bar{\lambda})$ ) of $\bar{\lambda}$, problem (2.4) has a nonempty solution set, that is, $S(\lambda) \neq \varnothing$ near $S(\bar{\lambda})$ and the setvalued mappings $S(\lambda)$ is continuous or Lipschitz continuous under the metric $\delta$ or $\tilde{H}$.

We need the following concepts and results.

Lemma 2.1 [5]. For each $x, v \in H$,

$$
x=P_{K}(v)
$$

if and only if

$$
\langle x-v, y-v\rangle \geq 0, \quad \forall y \in K,
$$

where $P_{K}(v)$ is the projection of $v \in H$ onto $K$.

LemMA 2.2 [9]. Let $m: H \rightarrow H$ be a single-valued mapping and

$$
K(x)=m(x)+K, \quad \forall x \in H .
$$

Then

$$
P_{K(x)}(y)=m(x)+P_{K}(y-m(x)), \quad \forall x, y \in H .
$$

Definition 2.3 [12]. A single-valued mapping $G: H \times \Omega \rightarrow H$ is called:

(i) $\alpha$-strongly monotone if there exists a constant $\alpha>0$ such that

$$
\left\langle G_{\lambda}(x)-G_{\lambda}(y), x-y\right\rangle \geq \alpha\|x-y\|^{2}, \quad \forall(x, y, \lambda) \in H \times H \times \Omega ;
$$


Salahuddin et al. 3

(ii) $\beta$-Lipschitz continuous if there exists a constant $\beta>0$ such that

$$
\left\|G_{\lambda}(x)-G_{\lambda}(y)\right\| \leq \beta\|x-y\|, \quad \forall(x, y, \lambda) \in H \times H \times \Omega .
$$

Definition 2.4 [1]. A set-valued mapping $R: H \times \Omega \rightarrow 2^{H}$ is said to be

(i) relaxed Lipschitz with respect to a mapping $f: H \times \Omega \rightarrow H$ if there exists a constant $r \geq 0$ such that

$$
\begin{gathered}
\left\langle f_{\lambda}\left(w_{\lambda}(x)\right)-f_{\lambda}\left(w_{\lambda}(y)\right), x-y\right\rangle \leq-r\|x-y\|^{2}, \\
\forall(x, y, \lambda) \in H \times H \times \Omega, \quad w_{\lambda}(x) \in R_{\lambda}(x), w_{\lambda}(y) \in R_{\lambda}(y) ;
\end{gathered}
$$

(ii) relaxed monotone with respect to a mapping $g: H \times \Omega \rightarrow H$ if there exists a constant $s>0$ such that

$$
\begin{gathered}
\left\langle g_{\lambda}\left(w_{\lambda}(x)\right)-g_{\lambda}\left(w_{\lambda}(y)\right), x-y\right\rangle \geq-s\|x-y\|^{2}, \\
\forall(x, y, \lambda) \in H \times H \times \Omega, \quad w_{\lambda}(x) \in R_{\lambda}(x), w_{\lambda}(y) \in R_{\lambda}(y) .
\end{gathered}
$$

Definition 2.5 [2]. A set-valued mapping $A: H \times \Omega \rightarrow 2^{H}[A: H \times \Omega \rightarrow C(H)]$ is said to be $\eta$ - $\delta$-Lipschitz $[\eta$ - $\tilde{H}$-Lipschitz ] continuous if there exists a constant $\eta \geq 0$ such that

$$
\begin{array}{ll}
\delta\left(A_{\lambda}(x), A_{\lambda}(y)\right) \leq \eta\|x-y\|, & \forall(x, y, \lambda) \in H \times H \times \Omega, \\
\tilde{H}\left(A_{\lambda}(x), A_{\lambda}(y)\right) \leq \eta\|x-y\|, & \forall(x, y, \lambda) \in H \times H \times \Omega .
\end{array}
$$

Lemma 2.6. Let $K_{\lambda}(x)$ be defined as (2.5). Then for each fixed $\bar{\lambda} \in \Omega$, problem (2.4) has a solution $\left(x(\bar{\lambda}), u_{\bar{\lambda}}(x(\bar{\lambda})), w_{\bar{\lambda}}(x(\bar{\lambda})), z_{\lambda}(x(\bar{\lambda}))\right)$ if and only if $\bar{x}=x(\bar{\lambda})$ is a fixed point of the set-valued mapping $\phi: H \times \Omega \rightarrow 2^{H}$ defined by

$$
\begin{aligned}
\phi_{\lambda}(x)= & \bigcup_{u_{\lambda}(x) \in A_{\lambda}(x), w_{\lambda}(x) \in R_{\lambda}(x), z_{\lambda}(x) \in T_{\lambda}(x)} \\
& {\left[x-G_{\lambda}(x)+m(x)\right.} \\
& \left.\quad+P_{K_{\lambda}}\left\{G_{\lambda}(x)-\rho\left(p_{\lambda}\left(u_{\lambda}(x)\right)-\left(f_{\lambda}\left(w_{\lambda}(x)\right)-g_{\lambda}\left(z_{\lambda}(x)\right)\right)\right)-m(x)\right\}\right],
\end{aligned}
$$

for each $x \in H$, where $\lambda=\bar{\lambda}, \rho>0$ is some constant and $P_{K_{\lambda}}(v)$ is the projection of $v \in H$ onto $K_{\bar{\lambda}}$.

Proof. For any fixed $\bar{\lambda} \in \Omega$, let $\left(\bar{x}, u_{\bar{\lambda}}(\bar{x}), w_{\bar{\lambda}}(\bar{x}), z_{\bar{\lambda}}(\bar{x})\right)$ be a solution of problem (2.4). Then $\bar{x} \in H, u_{\bar{\lambda}}(\bar{x}) \in A_{\bar{\lambda}}(\bar{x}), w_{\bar{\lambda}}(\bar{x}) \in R_{\bar{\lambda}}(\bar{x})$ and $z_{\bar{\lambda}}(\bar{x}) \in T_{\bar{\lambda}}(\bar{x})$ such that $G_{\bar{\lambda}}(\bar{x}) \in K_{\bar{\lambda}}(\bar{x})$ and

$$
\left\langle p_{\bar{\lambda}}\left(u_{\bar{\lambda}}(\bar{x})\right)-\left(f_{\bar{\lambda}}\left(w_{\bar{\lambda}}(\bar{x})\right)-g_{\bar{\lambda}}\left(z_{\bar{\lambda}}(\bar{x})\right)\right), y-G_{\bar{\lambda}}(\bar{x})\right\rangle \geq 0, \quad \forall y \in K_{\bar{\lambda}}(\bar{x}) .
$$

Hence for any $\rho>0$,

$$
\begin{aligned}
\left\langle G_{\bar{\lambda}}(\bar{x})-\left[G_{\bar{\lambda}}(\bar{x})-\rho\left(p_{\bar{\lambda}}\left(u_{\bar{\lambda}}(\bar{x})\right)-\left(f_{\bar{\lambda}}\left(w_{\bar{\lambda}}(\bar{x})\right)\right.\right.\right.\right. & \\
& \left.\left.\left.\left.-g_{\bar{\lambda}}\left(z_{\bar{\lambda}}(\bar{x})\right)\right)\right)\right], y-G_{\bar{\lambda}}(\bar{x})\right\rangle \geq 0, \quad \forall y \in K_{\bar{\lambda}}(\bar{x}) .
\end{aligned}
$$


4 Parametric problem of quasi-variational inequalities

From Lemmas 2.1 and 2.2, we have

$$
\begin{aligned}
G_{\bar{\lambda}}(\bar{x}) & =P_{K_{\bar{\lambda}}(\bar{x})}\left[G_{\bar{\lambda}}(\bar{x})-\rho\left(p_{\bar{\lambda}}\left(u_{\bar{\lambda}}(\bar{x})\right)-\left(f_{\bar{\lambda}}\left(w_{\bar{\lambda}}(\bar{x})\right)-g_{\bar{\lambda}}\left(z_{\bar{\lambda}}(\bar{x})\right)\right)\right)\right] \\
& =m(\bar{x})+P_{K_{\bar{\lambda}}}\left[G_{\bar{\lambda}}(\bar{x})-\rho\left(p_{\bar{\lambda}}\left(u_{\bar{\lambda}}(\bar{x})\right)-\left(f_{\bar{\lambda}}\left(w_{\bar{\lambda}}(\bar{x})\right)-g_{\bar{\lambda}}\left(z_{\bar{\lambda}}(\bar{x})\right)\right)\right)-m(\bar{x})\right] .
\end{aligned}
$$

We can also write

$$
\begin{aligned}
\bar{x}= & \bar{x}-G_{\bar{\lambda}}(\bar{x})+m(\bar{x}) \\
& +P_{K_{\bar{\lambda}}}\left[G_{\bar{\lambda}}(\bar{x})-\rho\left(p_{\bar{\lambda}}\left(u_{\bar{\lambda}}(\bar{x})\right)-\left(f_{\bar{\lambda}}\left(w_{\bar{\lambda}}(\bar{x})\right)-g_{\bar{\lambda}}\left(z_{\bar{\lambda}}(\bar{x})\right)\right)\right)-m(\bar{x})\right] \\
\in & \\
& u_{\bar{\lambda}}(\bar{x}) \in A_{\bar{\lambda}}(\bar{x}), w_{\bar{\lambda}}(\bar{x}) \in R_{\bar{\lambda}}(\bar{x}), z_{\bar{\lambda}}(\bar{x}) \in T_{\bar{\lambda}}(\bar{x}) \\
& \quad\left[\bar{x}-G_{\bar{\lambda}}(\bar{x})+m(\bar{x})\right. \\
& \left.\quad+P_{K_{\bar{\lambda}}}\left\{G_{\bar{\lambda}}(\bar{x})-\rho\left(p_{\bar{\lambda}}\left(u_{\bar{\lambda}}(\bar{x})\right)-\left(f_{\bar{\lambda}}\left(w_{\bar{\lambda}}(\bar{x})\right)-g_{\bar{\lambda}}\left(z_{\bar{\lambda}}(\bar{x})\right)\right)\right)-m(\bar{x})\right\}\right]=\phi_{\bar{\lambda}}(\bar{x}),
\end{aligned}
$$

that is, $\bar{x}=x(\bar{\lambda})$ is a fixed point of $\phi_{\bar{\lambda}}(\bar{x})$.

Now, for any fixed $\bar{\lambda} \in \Omega$, let $x(\bar{\lambda})$ be a fixed point of $\phi_{\bar{\lambda}}(\bar{x})$. By Lemma 2.1 there exist $u_{\bar{\lambda}}(\bar{x}) \in A_{\bar{\lambda}}(\bar{x}), w_{\bar{\lambda}}(\bar{x}) \in R_{\bar{\lambda}}(\bar{x})$ and $z_{\bar{\lambda}}(\bar{x}) \in T_{\bar{\lambda}}(\bar{x})$ such that

$$
\begin{aligned}
G_{\bar{\lambda}}(\bar{x}) & =m(\bar{x})+P_{K_{\bar{\lambda}}}\left[G_{\bar{\lambda}}(\bar{x})-\rho\left(p_{\bar{\lambda}}\left(u_{\bar{\lambda}}(\bar{x})\right)-\left(f_{\bar{\lambda}}\left(w_{\bar{\lambda}} \quad(\bar{x})\right)-g_{\bar{\lambda}}\left(z_{\bar{\lambda}}(\bar{x})\right)\right)\right)-m(\bar{x})\right] \\
& =P_{K_{\bar{\lambda}}(\bar{x})}\left[G_{\bar{\lambda}}(\bar{x})-\rho\left(p_{\bar{\lambda}}\left(u_{\bar{\lambda}}(\bar{x})\right)-\left(f_{\bar{\lambda}}\left(w_{\bar{\lambda}}(\bar{x})\right)-g_{\bar{\lambda}}\left(z_{\bar{\lambda}}(\bar{x})\right)\right)\right)\right] .
\end{aligned}
$$

Hence, we have $G_{\bar{\lambda}}(\bar{x}) \in K_{\bar{\lambda}}(\bar{x})$ and

$$
\left\langle G_{\bar{\lambda}}(\bar{x})-\left[G_{\bar{\lambda}}(\bar{x})-\rho\left(p_{\bar{\lambda}}\left(u_{\bar{\lambda}}(\bar{x})\right)-\left(f_{\bar{\lambda}}\left(w_{\bar{\lambda}}(\bar{x})\right)-g_{\bar{\lambda}}\left(z_{\bar{\lambda}}(\bar{x})\right)\right)\right)\right], y-G_{\bar{\lambda}}(\bar{x})\right\rangle \geq 0,
$$

for all $y \in K_{\bar{\lambda}}(\bar{x})$.

Noting that $\rho>0$, we have

$$
\left\langle p_{\bar{\lambda}}\left(u_{\bar{\lambda}}(\bar{x})\right)-\left(f_{\bar{\lambda}}\left(w_{\bar{\lambda}}(\bar{x})\right)-g_{\bar{\lambda}}\left(z_{\bar{\lambda}}(\bar{x})\right)\right), y-G_{\bar{\lambda}}(\bar{x})\right\rangle \geq 0, \quad \forall y \in K_{\bar{\lambda}}(\bar{x}),
$$

that is, $\left(\bar{x}, u_{\bar{\lambda}}(\bar{x}), w_{\bar{\lambda}}(\bar{x}), z_{\bar{\lambda}}(\bar{x})\right)$ is a solution of the problem (2.4).

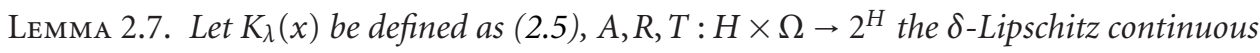
with respect to constants $\eta, \gamma, \nu$, respectively, and $p, f, g, G: H \times \Omega \rightarrow H$ the Lipschitz continuous with respect to the constants $\xi, \chi, \sigma$ and $\beta$, respectively. Let $G$ be strongly monotone with constant $\alpha>0, R$ relaxed Lipschitz continuous with respect to $f$ with constant $r \geq 0, T$ 
relaxed monotone with respect to $g$ with constant $s>0$, and $m: H \rightarrow H$ is $\mu$-Lipschitz continuous. If there exists a constant $\rho>0$ such that

$$
\begin{gathered}
\left|\rho-\frac{(r-s)+\xi \eta(q-1)}{(\gamma \chi+\sigma \nu)^{2}-(\xi \eta)^{2}}\right|<\frac{\sqrt{((r-s)+\xi \eta(q-1))^{2}-q(q-1)\left((\gamma \chi+\sigma \nu)^{2}-(\xi \eta)^{2}\right)}}{(\gamma \chi+\sigma \nu)^{2}-(\xi \eta)^{2}} \\
(r-s)>(1-q) \xi \eta+\sqrt{q(q-1)\left((\gamma \chi+\sigma \nu)^{2}-(\xi \eta)^{2}\right)} \\
\rho \xi \eta<\gamma \chi+\sigma \nu, \\
q=2\left(\mu+\sqrt{1-2 \alpha+\beta^{2}}\right)<1,
\end{gathered}
$$

then the set-valued mapping $\phi: H \times \Omega \rightarrow 2^{H}$ defined by (2.15) is a uniform $\theta$ - $\delta$-set-valued contraction with respect to $\lambda \in \Omega$, where

$$
\begin{gathered}
\theta=q+t(\rho)+\rho \xi \eta<1, \\
t(\rho)=\sqrt{1-2 \rho(r-s)+\rho^{2}(\gamma \chi+\sigma \nu)^{2}} .
\end{gathered}
$$

Proof. By the definition of $\phi$, for any $x, y \in H, \lambda \in \Omega, a \in \phi_{\lambda}(x)$ and $b \in \phi_{\lambda}(y)$, there exist $u_{\lambda}(x) \in A_{\lambda}(x), u_{\lambda}(y) \in A_{\lambda}(y), w_{\lambda}(x) \in R_{\lambda}(x), w_{\lambda}(y) \in R_{\lambda}(y), z_{\lambda}(x) \in T_{\lambda}(x)$ and $z_{\lambda}(y) \in T_{\lambda}(y)$ such that

$$
\begin{aligned}
& a=x-G_{\lambda}(x)+m(x)+P_{K_{\lambda}}\left[G_{\lambda}(x)-\rho\left(p_{\lambda}\left(u_{\lambda}(x)\right)-\left(f_{\lambda}\left(w_{\lambda}(x)\right)-g_{\lambda}\left(z_{\lambda}(x)\right)\right)\right)-m(x)\right], \\
& b=y-G_{\lambda}(y)+m(y)+P_{K_{\lambda}}\left[G_{\lambda}(y)-\rho\left(p_{\lambda}\left(u_{\lambda}(y)\right)-\left(f_{\lambda}\left(w_{\lambda}(y)\right)-g_{\lambda}\left(z_{\lambda}(y)\right)\right)\right)-m(y)\right] .
\end{aligned}
$$

Since projection operator is nonexpansive, we have

$$
\begin{aligned}
\|a-b\| \leq & 2\left\|x-y-\left(G_{\lambda}(x)-G_{\lambda}(y)\right)\right\|+2\|m(x)-m(y)\| \\
& +\left\|x-y+\rho\left(f_{\lambda}\left(w_{\lambda}(y)\right)-f_{\lambda}\left(w_{\lambda}(y)\right)\right)-\rho\left(g_{\lambda}\left(z_{\lambda}(x)\right)-g_{\lambda}\left(z_{\lambda}(y)\right)\right)\right\| \\
& +\rho\left\|p_{\lambda}\left(u_{\lambda}(x)\right)-p_{\lambda}\left(u_{\lambda}(y)\right)\right\| .
\end{aligned}
$$

Since $G$ is strongly monotone and Lipschitz continuous, we have

$$
\begin{gathered}
\left\|x-y-\left(G_{\lambda}(x)-G_{\lambda}(y)\right)\right\|^{2} \leq\left(1-2 \alpha+\beta^{2}\right)\|x-y\|^{2}, \\
\|m(x)-m(y)\| \leq \mu\|x-y\|, \\
\left\|p_{\lambda}\left(\mu_{\lambda}(x)\right)-p_{\lambda}\left(u_{\lambda}(y)\right)\right\| \leq \xi\left\|u_{\lambda}(x)-u_{\lambda}(y)\right\| \leq \xi \delta\left(A_{\lambda}(x), A_{\lambda}(y)\right) \leq \xi \eta\|x-y\| .
\end{gathered}
$$


6 Parametric problem of quasi-variational inequalities

Again

$$
\begin{aligned}
\| x-y+ & \rho\left(f_{\lambda}\left(w_{\lambda}(x)\right)-f_{\lambda}\left(w_{\lambda}(y)\right)\right)-\rho\left(g_{\lambda}\left(z_{\lambda}(x)\right)-g_{\lambda}\left(z_{\lambda}(y)\right)\right) \|^{2} \\
= & \|x-y\|^{2}+2 \rho\left\langle f_{\lambda}\left(w_{\lambda}(x)\right)-f_{\lambda}\left(w_{\lambda}(y)\right), x-y\right\rangle-2 \rho\left\langle g_{\lambda}\left(z_{\lambda}(x)\right)-g_{\lambda}\left(z_{\lambda}(y)\right), x-y\right\rangle \\
& +\rho^{2}\left\|f_{\lambda}\left(w_{\lambda}(x)\right)-f_{\lambda}\left(w_{\lambda}(y)\right)-\left(g_{\lambda}\left(z_{\lambda}(x)\right)-g_{\lambda}\left(z_{\lambda}(y)\right)\right)\right\|^{2} \\
\leq & {\left[1-2 \rho(r-s)+\rho^{2}(\gamma \chi+\sigma \nu)^{2}\right]\|x-y\|^{2} . }
\end{aligned}
$$

From (2.26)-(2.28), we have

$$
\|a-b\| \leq[q+t(\rho)+\rho \xi \eta]\|x-y\| \leq \theta\|x-y\|,
$$

where

$$
\begin{gathered}
\theta=q+t(\rho)+\rho \xi \eta, \\
t(\rho)=\sqrt{1-2 \rho(r-s)+\rho^{2}(\gamma \chi+\rho \nu)^{2}}, \\
q=2\left(\mu+\sqrt{1-2 \alpha+\beta^{2}}\right) .
\end{gathered}
$$

By the arbitrariness of $a$ and $b$, we have

$$
\delta\left(\phi_{\lambda}(x), \phi_{\lambda}(y)\right) \leq \theta d(x, y) .
$$

By conditions (2.23) and (2.24), we have $\theta<1$. This proves that $\theta$ is a uniform $\theta-\delta$-setvalued contraction with respect to $\lambda \in \Omega$.

Lemma 2.8 [6]. Let $X$ be a complete metric space and $T_{1}, T_{2}: X \rightarrow C(X)$ be $\theta-\tilde{H}$-contraction mapping. Then

$$
\tilde{H}\left(F\left(T_{1}\right), F\left(T_{2}\right)\right) \leq\left[\frac{1}{1-\theta}\right] \sup _{x \in X} \tilde{H}\left(T_{1}(x), T_{2}(x)\right),
$$

where $F\left(T_{1}\right)$ and $F\left(T_{2}\right)$ are the sets of fixed points of $T_{1}$ and $T_{2}$, respectively.

\section{Sensitivity analysis}

Theorem 3.1. Assume that $A_{\lambda}(\bar{x}), R_{\lambda}(\bar{x})$ and $T_{\lambda}(\bar{x})$ are $\delta$-Lipschitz continuous at $\bar{\lambda}$. Let $R_{\lambda}(\bar{x})$ be the relaxed Lipschitz continuous with $f_{\lambda}(\cdot)$ at $\bar{\lambda}$, and $T_{\lambda}(\bar{x})$ the relaxed monotone with $g_{\lambda}(\cdot)$ at $\bar{\lambda}$. Suppose that $G_{\lambda}(\bar{x}), p_{\lambda}(\cdot), f_{\lambda}(\cdot), g_{\lambda}(\cdot)$ and $P_{K_{\lambda}}(v)$ are Lipschitz continuous at $\bar{\lambda}$, where $\bar{x}=\bar{x}(\bar{\lambda}) \in S(\bar{\lambda}), \bar{u}_{\bar{\lambda}}(\bar{x}) \in A_{\bar{\lambda}}(\bar{x}), \bar{w}_{\bar{\lambda}}(\bar{x}) \in R_{\bar{\lambda}}(\bar{x}), \bar{z}_{\bar{\lambda}}(\bar{x}) \in T_{\bar{\lambda}}(\bar{x})$ and

$$
v=G_{\bar{\lambda}}(\bar{x})-\rho\left(p_{\bar{\lambda}}\left(\bar{u}_{\bar{\lambda}}(\bar{x})\right)-\left(f_{\bar{\lambda}}\left(\bar{w}_{\bar{\lambda}}(\bar{x})\right)-g_{\bar{\lambda}}\left(\bar{z}_{\bar{\lambda}}(\bar{x})\right)\right)\right)-m(\bar{x}) .
$$

Then for all $\lambda \in \Omega$, the solution set $S(\lambda)$ of the problem (2.4) is nonempty and $S(\lambda)$ is $\delta$ Lipschitz continuous at $\bar{\lambda}$.

Proof. For each fixed $\lambda \in \Omega, \phi_{\lambda}(x)$ has a fixed point, that is, there exists a $x(\bar{\lambda}) \in H$ such that $x(\lambda) \in \phi_{\lambda}(x(\lambda))$. From Lemma 2.6, we have $x(\lambda) \in S(\lambda)$, hence $S(\lambda) \neq \varnothing$ and $S(\lambda)$ coincides with the set of fixed point of $\phi_{\lambda}(x)$. In particular, $S(\bar{\lambda})$ coincides with the set of 
fixed point of $\phi_{\bar{\lambda}}(x)$. Now we show that $S(\lambda)$ is $\delta$-Lipschitz continuous at $\bar{\lambda}$. For all $x(\lambda) \in$ $S(\lambda)$ and $\bar{x}(\bar{\lambda}) \in S(\bar{\lambda})$ there exist $u_{\lambda}(x(\lambda)) \in A_{\lambda}(x(\lambda)), w_{\lambda}(x(\lambda)) \in R_{\lambda}(x(\lambda)), z_{\lambda}(x(\lambda)) \in$ $T_{\lambda}(x(\lambda)), \bar{u}_{\bar{\lambda}}(\bar{x}(\bar{\lambda})) \in A_{\bar{\lambda}}(\bar{x}(\bar{\lambda})), \bar{w}_{\bar{\lambda}}(\bar{x}(\bar{\lambda})) \in R_{\bar{\lambda}}(\bar{x}(\bar{\lambda}))$ and $\bar{z}_{\bar{\lambda}}(\bar{x}(\bar{\lambda})) \in T_{\bar{\lambda}}(\bar{x}(\bar{\lambda}))$ such that

$$
\begin{aligned}
x(\lambda)= & x(\lambda)-G_{\lambda}(x(\lambda))+m(x(\lambda)) \\
& +P_{K_{\lambda}}\left[G_{\lambda}(x(\lambda))-\rho\left(p_{\lambda}\left(u_{\lambda}(x(\lambda))\right)-\left(f_{\lambda}\left(w_{\lambda}(x(\lambda))\right)-g_{\lambda}\left(z_{\lambda}(x(\lambda))\right)\right)\right)-m(x(\lambda))\right], \\
\bar{x}(\bar{\lambda})= & \bar{x}(\bar{\lambda})-G_{\bar{\lambda}}(\bar{x}(\bar{\lambda}))+m(\bar{x}(\bar{\lambda})) \\
& +P_{K_{\bar{\lambda}}}\left[G_{\bar{\lambda}}(\bar{x}(\bar{\lambda}))-\rho\left(p_{\bar{\lambda}}\left(\bar{u}_{\bar{\lambda}}(\bar{x}(\bar{\lambda}))\right)-\left(f_{\bar{\lambda}}\left(\bar{w}_{\bar{\lambda}}(\bar{x}(\bar{\lambda}))\right)-g_{\bar{\lambda}}\left(\bar{z}_{\bar{\lambda}}(\bar{x}(\bar{\lambda}))\right)\right)\right)-m(\bar{x}(\bar{\lambda}))\right] .
\end{aligned}
$$

Write $x=x(\lambda)$ and $\bar{x}=\bar{x}(\bar{\lambda})$. Taking any $u_{\lambda}(\bar{x}) \in A_{\lambda}(\bar{x}), w_{\lambda}(\bar{x}) \in R_{\lambda}(\bar{x})$ and $z_{\lambda}(\bar{x}) \in$ $T_{\lambda}(\bar{x})$, we have

$$
\begin{aligned}
\|x-\bar{x}\| \leq & \| x-G_{\lambda}(x)+m(x) \\
+ & P_{K_{\lambda}}\left[G_{\lambda}(x)-\rho\left(p_{\lambda}\left(u_{\lambda}(x)\right)-\left(f_{\lambda}\left(w_{\lambda}(x)\right)-g_{\lambda}\left(z_{\lambda}(x)\right)\right)\right)-m(x)\right] \\
- & {\left[\bar{x}-G_{\lambda}(\bar{x})+m(\bar{x})\right.} \\
& \left.+P_{K_{\lambda}}\left\{G_{\lambda}(\bar{x})-\rho\left(p_{\lambda}\left(u_{\lambda}(\bar{x})\right)-\left(f_{\lambda}\left(w_{\lambda}(\bar{x})\right)-g_{\lambda}\left(z_{\lambda}(\bar{x})\right)\right)\right)-m(\bar{x})\right\}\right] \| \\
+ & \| \bar{x}-G_{\lambda}(\bar{x})+m(\bar{x}) \\
& +P_{K_{\lambda}}\left[G_{\lambda}(\bar{x})-\rho\left(p_{\lambda}\left(u_{\lambda}(\bar{x})\right)-\left(f_{\lambda}\left(w_{\lambda}(\bar{x})\right)-g_{\lambda}\left(z_{\lambda}(\bar{x})\right)\right)\right)-m(\bar{x})\right] \\
& -\left[\bar{x}-G_{\bar{\lambda}}(\bar{x})+m(\bar{x})\right. \\
& \left.\quad+P_{K_{\bar{\lambda}}}\left\{G_{\bar{\lambda}}(\bar{x})-\rho\left(p_{\bar{\lambda}}\left(\bar{u}_{\bar{\lambda}}(\bar{x})\right)-\left(f_{\bar{\lambda}}\left(\bar{w}_{\bar{\lambda}}(\bar{x})\right)-g_{\bar{\lambda}}\left(\bar{z}_{\bar{\lambda}}(\bar{x})\right)\right)\right)-m(\bar{x})\right\}\right] \| \\
\leq & \theta\|x-\bar{x}\|+\left\|G_{\lambda}(\bar{x})-G_{\bar{\lambda}}(\bar{x})\right\| \\
+ & \| P_{K_{\lambda}}\left[G_{\lambda}(\bar{x})-\rho\left(p_{\lambda}\left(u_{\lambda}(\bar{x})\right)-\left(f_{\lambda}\left(w_{\lambda}(\bar{x})\right)-g_{\lambda}\left(z_{\lambda}(\bar{x})\right)\right)\right)-m(\bar{x})\right] \\
& \quad-P_{K_{\lambda}}\left[G_{\bar{\lambda}}(\bar{x})-\rho\left(p_{\bar{\lambda}}\left(\bar{u}_{\bar{\lambda}}(\bar{x})\right)-\left(f_{\bar{\lambda}}\left(\bar{w}_{\bar{\lambda}}(\bar{x})\right)-g_{\bar{\lambda}}\left(\bar{z}_{\bar{\lambda}}(\bar{x})\right)\right)\right)-m(\bar{x})\right] \| \\
+ & \| P_{K_{\lambda}}\left[G_{\bar{\lambda}}(\bar{x})-\rho\left(p_{\bar{\lambda}}\left(\bar{u}_{\bar{\lambda}}(\bar{x})\right)-\left(f_{\bar{\lambda}}\left(\bar{w}_{\bar{\lambda}}(\bar{x})\right)-g_{\bar{\lambda}}\left(\bar{z}_{\bar{\lambda}}(\bar{x})\right)\right)\right)-m(\bar{x})\right] \\
& \quad-P_{K_{\bar{\lambda}}}\left[G_{\bar{\lambda}}(\bar{x})-\rho\left(p_{\bar{\lambda}}\left(\bar{u}_{\bar{\lambda}}(\bar{x})\right)-\left(f_{\bar{\lambda}}\left(\bar{w}_{\bar{\lambda}}(\bar{x})\right)-g_{\bar{\lambda}}\left(\bar{z}_{\bar{\lambda}}(\bar{x})\right)\right)\right)-m(\bar{x})\right] \| \\
\leq & \theta\|x-\bar{x}\|+2\left\|G_{\lambda}(\bar{x})-G_{\bar{\lambda}}(\bar{x})\right\|+\rho\left\|p_{\lambda}\left(u_{\lambda}(\bar{x})\right)-p_{\bar{\lambda}}\left(\bar{u}_{\bar{\lambda}}(\bar{x})\right)\right\| \\
+ & \rho\left\|f_{\lambda}\left(w_{\lambda}(\bar{x})\right)-f_{\bar{\lambda}}\left(\bar{w}_{\bar{\lambda}}(\bar{x})\right)\right\| \\
+ & \rho\left\|g_{\lambda}\left(z_{\lambda}(\bar{x})\right)-g_{\bar{\lambda}}\left(\bar{z}_{\bar{\lambda}}(\bar{x})\right)\right\|+\left\|P_{K_{\lambda}}(v)-P_{k_{\bar{\lambda}}}(v)\right\|,
\end{aligned}
$$

where, $v=G_{\bar{\lambda}}(\bar{x})-\rho\left(p \bar{\lambda}\left(\bar{u}_{\bar{\lambda}}(\bar{x})\right)-\left(f_{\bar{\lambda}}\left(\bar{w}_{\bar{\lambda}}(\bar{x})\right)-g_{\bar{\lambda}}\left(\bar{z}_{\bar{\lambda}}(\bar{x})\right)\right)\right)-m(\bar{x})$. Since, $x=x(\lambda) \in S(\lambda)$ and $\bar{x}=\bar{x}(\bar{\lambda}) \in S(\bar{\lambda})$ are arbitrary, it follows that

$$
\begin{aligned}
\delta(S(\lambda), S(\bar{\lambda})) \leq\left[\frac{1}{1-\theta}\right][ & {\left[2\left\|G_{\lambda}(\bar{x})-G_{\bar{\lambda}}(\bar{x})\right\|+\rho\left\|p_{\lambda}\left(u_{\lambda}(\bar{x})\right)-p_{\bar{\lambda}}\left(\bar{u}_{\bar{\lambda}}(\bar{x})\right)\right\|+\rho \| f_{\lambda}\left(w_{\lambda}(\bar{x})\right)\right.} \\
& \left.-f_{\bar{\lambda}}\left(\bar{w}_{\bar{\lambda}}(\bar{x})\right)\|+\rho\| g_{\lambda}\left(z_{\lambda}(\bar{x})\right)-g_{\bar{\lambda}}\left(\bar{z}_{\bar{\lambda}}(\bar{x})\right)\|+\| P_{K_{\lambda}}(v)-P_{K_{\bar{\lambda}}}(v) \|\right] .
\end{aligned}
$$


8 Parametric problem of quasi-variational inequalities

From the $\delta$-Lipschitz continuity of $A, R, T$ at $\bar{\lambda}$; Lipschitz continuity of $G$ and $P_{K_{\lambda}}(v)$ at $\bar{\lambda}$, it follows that $S(\lambda)$ is $\delta$-Lipschitz continuous.

THeOREм 3.2. If we assume the hypothesis of Lemma 2.7, then

(i) $\phi: H \times \Omega \rightarrow C(H)$ defined by (2.15) is a compact valued uniform $\theta-\tilde{H}$-contraction mapping with respect to $\lambda \in \Omega$;

(ii) for each $\lambda \in \Omega,(2.4)$ has nonempty solution set $S(\lambda)$, closed in $H$.

Proof. (i) For each $(x, \lambda) \in H \times \Omega ; A_{\lambda}(x), R_{\lambda}(x), T_{\lambda}(x) \in C(H)$ and $P_{K_{\lambda}}$ are continuous, follows from (2.15) of $\phi_{\lambda}(x) \in C(H)$. Now, we show that $\phi_{\lambda}(x)$ is a uniform $\theta$ $\tilde{H}$-contraction mapping with respect to $\lambda \in \Omega$. For any $a \in \phi_{\lambda}(x)$, there exist $u_{\lambda}(x) \in$ $A_{\lambda}(x) \in C(H), w_{\lambda}(x) \in R_{\lambda}(x) \in C(H)$ and $z_{\lambda}(x) \in T_{\lambda}(x) \in C(H)$ such that

$$
a=x-G_{\lambda}(x)+m(x)+P_{K_{\lambda}}\left[G_{\lambda}(x)-\rho\left(p_{\lambda}\left(u_{\lambda}(x)\right)-\left(f_{\lambda}\left(w_{\lambda}(x)\right)-g_{\lambda}\left(z_{\lambda}(x)\right)\right)\right)-m(x)\right] .
$$

Note that $(y, \lambda) \in H \times \Omega ; A_{\lambda}(y), R_{\lambda}(y), T_{\lambda}(y) \in C(H)$, then there exist $u_{\lambda}(y) \in A_{\lambda}(y)$, $w_{\lambda}(y) \in R_{\lambda}(y)$ and $z_{\lambda}(y) \in T_{\lambda}(y)$ such that

$$
\begin{gathered}
\left\|p_{\lambda}\left(u_{\lambda}(x)\right)-p_{\lambda}\left(u_{\lambda}(y)\right)\right\| \leq \xi\left\|u_{\lambda}(x)-u_{\lambda}(y)\right\| \leq \xi \tilde{H}\left(A_{\lambda}(x), A_{\lambda}(y)\right), \\
\left\|f_{\lambda}\left(w_{\lambda}(x)\right)-f_{\lambda}\left(w_{\lambda}(y)\right)\right\| \leq \chi\left\|w_{\lambda}(x)-w_{\lambda}(y)\right\| \leq \chi \tilde{H}\left(R_{\lambda}(x), R_{\lambda}(y)\right), \\
\left\|g_{\lambda}\left(z_{\lambda}(x)\right)-g_{\lambda}\left(z_{\lambda}(y)\right)\right\| \leq \sigma\left\|z_{\lambda}(x)-z_{\lambda}(y)\right\| \leq \sigma \tilde{H}\left(T_{\lambda}(x), T_{\lambda}(y)\right) .
\end{gathered}
$$

Let

$$
b=y-G_{\lambda}(y)+m(y)+P_{K_{\lambda}}\left[G_{\lambda}(y)-\rho\left(p_{\lambda}\left(u_{\lambda}(y)\right)-\left(f_{\lambda}\left(w_{\lambda}(y)\right)-g_{\lambda}\left(z_{\lambda}(y)\right)\right)\right)-m(y)\right],
$$

then

$$
b \in \phi_{\lambda}(y) .
$$

By using the similar argument as in the proof of Lemma 2.7, we can obtain

$$
\begin{aligned}
\|a-b\| & \leq\left[2\left(\mu+\sqrt{1-2 \alpha+\beta^{2}}\right)+\sqrt{1-2 \rho(r-s)+\rho^{2}(\gamma \chi+\sigma \nu)^{2}}+\rho \xi \eta\right]\|x-y\| \\
& \leq[q+t(\rho)+\rho \xi \eta]\|x-y\| \leq \theta\|x-y\|,
\end{aligned}
$$

where

$$
\begin{gathered}
\theta=q+t(\rho)+\rho \xi \eta, \\
t(\rho)=\sqrt{1-2 \rho(r-s)+\rho^{2}(\gamma \chi+\sigma \nu)^{2}}, \\
q=2\left(\mu+\sqrt{1-2 \alpha+\beta^{2}}\right) .
\end{gathered}
$$


By conditions (2.23) and (2.24), $\theta<1$, and hence we have

$$
\sup _{a \in \phi_{\lambda}(x)} d\left(a, \phi_{\lambda}(y)\right) \leq \theta\|x-y\|
$$

By the similar arguments, we have

$$
\sup _{b \in \phi_{\lambda}(y)} d\left(\phi_{\lambda}(x), b\right) \leq \theta\|x-y\| \text {. }
$$

Hence, by the Hausdorff metric $\tilde{H}$, we obtain

$$
\tilde{H}\left(\phi_{\lambda}(x), \phi_{\lambda}(y)\right) \leq \theta\|x-y\| \text {. }
$$

Therefore $\phi_{\lambda}(x)$ is a uniform $\theta-\tilde{H}$-contraction mapping with respect to $\lambda \in \Omega$.

(ii) Since $\phi_{\lambda}(x)$ is a uniform $\theta-\tilde{H}$-contraction with respect to $\lambda \in \Omega$, hence by Nadler theorem [8], $\phi_{\lambda}(x)$ has a fixed point $x(\lambda)$. Since $S(\lambda) \neq \varnothing$, then let $\left\{x_{n}\right\} \subset S(\lambda)$ and $x_{n} \rightarrow$ $x_{0}$ as $n \rightarrow \infty$. Therefore,

$$
x_{n} \in \phi_{\lambda}\left(x_{n}\right), \quad n=1,2, \ldots
$$

From (i), we have

$$
\tilde{H}\left(\phi_{\lambda}\left(x_{n}\right), \phi_{\lambda}\left(x_{0}\right)\right) \leq \theta\left\|x_{n}-x_{0}\right\| \text {. }
$$

If follows that

$$
\begin{aligned}
d\left(x_{0}, \phi_{\lambda}\left(x_{0}\right)\right) & \leq\left\|x_{0}-x_{n}\right\|+d\left(x_{n}, \phi_{\lambda}\left(x_{n}\right)\right)+\tilde{H}\left(\phi_{\lambda}\left(x_{n}\right), \phi_{\lambda}\left(x_{0}\right)\right) \\
& \leq(1+\theta)\left\|x_{n}-x_{0}\right\| \longrightarrow 0, \quad \text { as } n \longrightarrow \infty,
\end{aligned}
$$

hence $x_{0} \in \phi_{\lambda}\left(x_{0}\right)$ and $x_{0} \in S(\lambda)$. Therefore $S(\lambda)$ is closed in $H$.

Theorem 3.3. Assume the hypothesis as in Theorem 3.1. Then for all $\lambda \in \Omega$, the solution set $S(\lambda)$ of (2.4) is nonempty and $S(\lambda)$ is $\tilde{H}$-Lipschitz continuous at $\bar{\lambda}$.

Proof. From Theorem 3.2(ii), the solution set $S(\lambda)$ of (2.4) is a nonempty closed set in $H$. Now, we show that $S(\lambda)$ is $\tilde{H}$-Lipschitz continuous at $\bar{\lambda}$. By Theorem 3.2(i), $\phi_{\lambda}(x)$ and $\phi_{\bar{\lambda}}(x)$ are both $\theta-\tilde{H}$-contraction mappings. From Lemma 2.8 , we have

$$
\tilde{H}(S(\lambda), S(\bar{\lambda})) \leq\left[\frac{1}{1-\theta}\right]_{x \in H} \sup _{x} \tilde{H}\left(\phi_{\lambda}(x), \phi_{\bar{\lambda}}(x)\right)
$$

Taking any $a \in \phi_{\lambda}(x), \exists u_{\lambda}(x) \in A_{\lambda}(x), w_{\lambda}(x) \in R_{\lambda}(x)$ and $z_{\lambda}(x) \in T_{\lambda}(x)$ such that

$$
a=x-G_{\lambda}(x)+m(x)+P_{K_{\lambda}}\left[G_{\lambda}(x)-\rho\left(p_{\lambda}\left(u_{\lambda}(x)\right)-\left(f_{\lambda}\left(w_{\lambda}(x)\right)-g_{\lambda}\left(z_{\lambda}(x)\right)\right)\right)-m(x)\right] .
$$


For $u_{\lambda}(x) \in A_{\lambda}(x) \in C(H), w_{\lambda}(x) \in R_{\lambda}(x) \in C(H), z_{\lambda}(x) \in T_{\lambda}(x) \in C(H)$, there exist $u_{\bar{\lambda}}(x) \in A_{\bar{\lambda}}(x), w_{\bar{\lambda}}(x) \in R_{\bar{\lambda}}(x)$ and $z_{\bar{\lambda}}(x) \in T_{\bar{\lambda}}(x)$ such that

$$
\begin{gathered}
\left\|u_{\lambda}(x)-u_{\bar{\lambda}}(x)\right\| \leq \tilde{H}\left(A_{\lambda}(x), A_{\bar{\lambda}}(x)\right), \\
\left\|w_{\lambda}(x)-w_{\bar{\lambda}}(x)\right\| \leq \tilde{H}\left(R_{\lambda}(x), R_{\bar{\lambda}}(x)\right), \\
\left\|z_{\lambda}(x)-z_{\bar{\lambda}}(x)\right\| \leq \tilde{H}\left(T_{\lambda}(x), T_{\bar{\lambda}}(x)\right) .
\end{gathered}
$$

Let

$$
b=x-G_{\bar{\lambda}}(x)+m(x)+P_{K_{\bar{\lambda}}}\left[G_{\bar{\lambda}}(x)-\rho\left(p_{\bar{\lambda}}\left(u_{\bar{\lambda}}(x)\right)-\left(f_{\bar{\lambda}}\left(w_{\bar{\lambda}}(x)\right)-g_{\bar{\lambda}}\left(z_{\bar{\lambda}}(x)\right)\right)\right)-m(x)\right]
$$

then

$$
b \in \phi_{\bar{\lambda}}(x)
$$

It follows that

$$
\begin{aligned}
\|a-b\| \leq & \left\|G_{\lambda}(x)-G_{\bar{\lambda}}(x)\right\| \\
& +\| P_{K_{\lambda}}\left\{G_{\lambda}(x)-\rho\left(p_{\lambda}\left(u_{\lambda}(x)\right)-\left(f_{\lambda}\left(w_{\lambda}(x)\right)-g_{\lambda}\left(z_{\lambda}(x)\right)\right)\right)-m(x)\right\} \\
& \quad-P_{K_{\lambda}}\left\{G_{\bar{\lambda}}(x)-\rho\left(p_{\bar{\lambda}}\left(u_{\bar{\lambda}}(x)\right)-\left(f_{\bar{\lambda}}\left(w_{\bar{\lambda}}(x)\right)-g_{\bar{\lambda}}\left(z_{\bar{\lambda}}(x)\right)\right)\right)-m(x)\right\} \| \\
+ & \| P_{K_{\lambda}}\left\{G_{\bar{\lambda}}(x)-\rho\left(p_{\bar{\lambda}}\left(u_{\bar{\lambda}}(x)\right)-\left(f_{\bar{\lambda}}\left(w_{\bar{\lambda}}(x)\right)-g_{\bar{\lambda}}\left(z_{\bar{\lambda}}(x)\right)\right)-m(x)\right\}\right. \\
& \quad-P_{K_{\bar{\lambda}}}\left\{G_{\bar{\lambda}}(x)-\rho\left(p_{\bar{\lambda}}\left(u_{\bar{\lambda}}(x)\right)-\left(f_{\bar{\lambda}}\left(w_{\bar{\lambda}}(x)\right)-g_{\bar{\lambda}}\left(z_{\bar{\lambda}}(x)\right)\right)\right)-m(x)\right\} \| \\
\leq & 2\left\|G_{\lambda}(x)-G_{\bar{\lambda}}(x)\right\|+\rho\left\|p_{\lambda}\left(u_{\lambda}(x)\right)-p_{\bar{\lambda}}\left(u_{\bar{\lambda}}(x)\right)\right\| \\
& +\rho\left\|f_{\lambda}\left(w_{\lambda}(x)\right)-f_{\bar{\lambda}}\left(w_{\bar{\lambda}}(x)\right)\right\|+\rho\left\|g_{\lambda}\left(z_{\lambda}(x)\right)-g_{\bar{\lambda}}\left(z_{\bar{\lambda}}(x)\right)\right\| \\
& +\left\|P_{K_{\lambda}}(v)-P_{K_{\bar{\lambda}}}(v)\right\| \leq 2\left\|G_{\lambda}(x)-G_{\bar{\lambda}}(x)\right\| \\
& +\rho\left\|p_{\lambda}\left(u_{\lambda}(x)\right)-p_{\bar{\lambda}}\left(u_{\lambda}(x)\right)\right\|+\rho\left\|p_{\bar{\lambda}}\left(u_{\lambda}(x)\right)-p_{\bar{\lambda}}\left(u_{\bar{\lambda}}(x)\right)\right\| \\
& +\rho\left\|f_{\lambda}\left(w_{\lambda}(x)\right)-f_{\bar{\lambda}}\left(w_{\lambda}(x)\right)\right\|+\rho\left\|f_{\bar{\lambda}}\left(w_{\lambda}(x)\right)-f_{\bar{\lambda}}\left(w_{\bar{\lambda}}(x)\right)\right\| \\
& +\rho\left\|g_{\lambda}\left(z_{\lambda}(x)\right)-g_{\bar{\lambda}}\left(z_{\lambda}(x)\right)\right\|+\rho\left\|g_{\bar{\lambda}}\left(z_{\lambda}(x)\right)-g_{\bar{\lambda}}\left(z_{\bar{\lambda}}(x)\right)\right\| \\
& +\left\|P_{K_{\lambda}}(v)-P_{K_{\bar{\lambda}}}(v)\right\|,
\end{aligned}
$$

where $v=G_{\bar{\lambda}}(x)-\rho\left(p_{\bar{\lambda}}\left(u_{\bar{\lambda}}(x)\right)-\left(f_{\bar{\lambda}}\left(w_{\bar{\lambda}}(x)\right)-g_{\bar{\lambda}}\left(z_{\bar{\lambda}}(x)\right)\right)\right)-m(x)$.

Write

$$
\begin{aligned}
M= & 2\left\|G_{\lambda}(x)-G_{\bar{\lambda}}(x)\right\|+\rho\left\|p_{\lambda}\left(u_{\lambda}(x)\right)-p_{\bar{\lambda}}\left(u_{\lambda}(x)\right)\right\| \\
& +\rho\left\|f_{\lambda}\left(w_{\lambda}(x)\right)-f_{\bar{\lambda}}\left(w_{\lambda}(x)\right)\right\|+\rho\left\|g_{\lambda}\left(z_{\lambda}(x)\right)-g_{\bar{\lambda}}\left(z_{\lambda}(x)\right)\right\| \\
& +\rho \xi \tilde{H}\left(A_{\lambda}(x), A_{\bar{\lambda}}(x)\right)+\rho \chi \tilde{H}\left(R_{\lambda}(x), R_{\bar{\lambda}}(x)\right)+\rho \sigma \tilde{H}\left(T_{\lambda}(x), T_{\bar{\lambda}}(x)\right) \\
& +\left\|P_{K_{\lambda}}(v)-P_{K_{\bar{\lambda}}}(v)\right\| .
\end{aligned}
$$


Then we have

$$
\sup _{a \in \phi_{\lambda}(x)} d\left(a, \phi_{\bar{\lambda}}(x)\right) \leq M .
$$

By the similar arguments, we obtain

$$
\sup _{b \in \phi_{\bar{\lambda}}(x)} d\left(\phi_{\lambda}(x), b\right) \leq M .
$$

It follows that

$$
\widetilde{H}\left(\phi_{\lambda}(x), \phi_{\bar{\lambda}}(x)\right) \leq M \text {. }
$$

If $A_{\lambda}(x), R_{\lambda}(x)$ and $T_{\lambda}(x)$ are uniformly $\tilde{H}$-Lipschitz continuous at $\bar{\lambda}$ with respect to $x \in$ $H$, and $G_{\lambda}(x), P_{K_{\lambda}}(v)$ are uniformly Lipschitz continuous at $\bar{\lambda}$ with respect to $x \in H$, then it follows that: for any $\epsilon>0$, there exists a $\delta>0$ such that for all $\lambda \in \Omega$ with $\|\lambda-\bar{\lambda}\|<\delta$,

$$
\tilde{H}\left(\phi_{\lambda}(x), \phi_{\bar{\lambda}}(x)\right) \leq M<\epsilon, \quad \forall x \in H .
$$

From (3.17), we obtain

$$
H(S(\lambda), S(\bar{\lambda}))<\frac{\epsilon}{1-\theta},
$$

hence $S(\lambda)$ is $\tilde{H}$-continuous at $\bar{\lambda}$. If $A_{\lambda}(x), R_{\lambda}(x)$ and $T_{\lambda}(x)$ are uniformly $\tilde{H}$-Lipschitz continuous at $\bar{\lambda}$ with respect to $x \in H$, and $G_{\lambda}(x), P_{K_{\lambda}}(v)$ are also uniformly Lipschitz continuous at $\bar{\lambda}$ with respect to $x \in H$, then by the above arguments, we can prove that $S(\lambda)$ is $\tilde{H}$-Lipschitz continuous.

\section{Acknowledgment}

A. H. Siddiqi would like to thank King Fahd University of Petroleum Minerals, Dhahran, Saudi Arabia, for providing excellent research environment.

\section{References}

[1] R. Ahmad, K. R. Kazmi, and Salahuddin, Completely generalized non-linear variational inclusions involving relaxed Lipschitz and relaxed monotone mappings, Nonlinear Analysis Forum 5 (2000), 61-69.

[2] R. W. Cottle, F. Giannessi, and J. L. Lions, Variational Inequalities and Complementarity Problems, Theory and Applications, John Wiley \& Sons, Chichester, 1980.

[3] S. Dafermos, Sensitivity analysis in variational inequalities, Mathematics of Operations Research 13 (1988), no. 3, 421-434.

[4] X. P. Ding and C. L. Luo, On parametric generalized quasi-variational inequalities, Journal of Optimization Theory and Applications 100 (1999), no. 1, 195-205.

[5] D. Kinderlehrer and G. Stampacchia, An Introduction to Variational Inequalities and Their Applications, Pure and Applied Mathematics, vol. 88, Academic Press, New York, 1980.

[6] T.-C. Lim, On fixed point stability for set-valued contractive mappings with applications to generalized differential equations, Journal of Mathematical Analysis and Applications 110 (1985), no. 2, 436-441. 


\section{Parametric problem of quasi-variational inequalities}

[7] R. N. Mukherjee and H. L. Verma, Sensitivity analysis of generalized variational inequalities, Journal of Mathematical Analysis and Applications 167 (1992), no. 2, 299-304.

[8] S. B. Nadler Jr., Multi-valued contraction mappings, Pacific Journal of Mathematics 30 (1969), no. $2,475-488$.

[9] M. A. Noor, An iterative scheme for a class of quasivariational inequalities, Journal of Mathematical Analysis and Applications 110 (1985), no. 2, 463-468.

[10] S. M. Robinson, Sensitivity analysis of variational inequalities by normal-map techniques, Variational Inequalities and Network Equilibrium Problems (Erice, 1994) (F. Giannessi and A. Maugeri, eds.), Plenum, New York, 1995, pp. 257-269.

[11] Salahuddin, Some aspects of variational inequalities, Ph.D. thesis, AMU, Aligarh, 2000.

[12] _ On parametric generalized quasivariational inequalities with related Lipschitz and relaxed monotone mappings, Advances in Nonlinear Variational Inequalities 5 (2002), no. 1, 107-114.

[13] A. H. Siddiqi and Q. H. Ansari, An algorithm for a class of quasivariational inequalities, Journal of Mathematical Analysis and Applications 145 (1990), no. 2, 413-418.

[14] N. D. Yen, Lipschitz continuity of solutions of variational inequalities with a parametric polyhedral constraint, Mathematics of Operations Research 20 (1995), no. 3, 695-708.

Salahuddin: Department of Mathematics, Aligarh Muslim University, Aligarh 202002 (UP), India E-mail address: salahuddin12@mailcity.com

M. K. Ahmad: Department of Mathematics, Aligarh Muslim University, Aligarh 202002 (UP), India E-mail address: ahmad_kalimuddin@yahoo.co.in

A. H. Siddiqi: Department of Mathematical Sciences, King Fahd University of Petroleum \& Minerals, P.O. Box 1745. Dhahran 31261, Saudi Arabia

E-mail address: ahasan@kfupm.edu.sa 